\title{
Parameter-free symmetry-preserving regularization modelling of turbulent natural convection flows
}

\author{
F.X. Trias, R.W.C.P. Verstappen, M. Soria and A. Oliva
}

\begin{abstract}
Since direct numerical simulations of natural convection flows cannot be performed at high $R a$-numbers, a dynamically less complex mathematical formulation is sought. In the quest for such a formulation, we consider regularizations (smooth approximations) of the nonlinearity. The regularization method basically alters the convective terms to reduce the production of small scales of motion by means of vortex stretching. In doing so, we propose to preserve the symmetry and conservation properties of the convective terms exactly. This requirement yields a novel class of regularizations that restrain the convective production of smaller and smaller scales of motion by means of vortex stretching in an unconditional stable manner, meaning that the velocity cannot blow up in the energy-norm (in 2D also: enstrophy-norm). The numerical algorithm used to solve the governing equations preserves the symmetry and conservation properties too. The regularization model is successfully tested for a 3D natural convection flow in air-filled $(\operatorname{Pr}=0.71)$ differentially heated cavity of height aspect ratio 4 at $R a=10^{10}$ and $10^{11}$. Moreover, a method to dynamically determine the regularization parameter (local filter length) is also proposed and tested.
\end{abstract}

\section{Introduction}

Turbulent natural convection in enclosed cavities has been the subject of numerous studies over the past decades. They model many engineering applications such as ventilation of rooms, cooling of electronics devices or air flow in buildings. Simulta-

F.X. Trias, M. Soria, A. Oliva

Centre Tecnològic de Transferència de Calor, ETSEIAT, Technical University of Catalonia, C/Colom 11, 08222 Terrassa, Spain, e-mail: cttc@cttc.upc.edu

F.X. Trias, R.W.C.P. Verstappen

Institute of Mathematics and Computing Science, University of Groningen, P.O. Box 407, 9700 AK Groningen, The Netherlands, e-mail: R.W.C.P.Verstappen@rug.nl 
neously, this configuration has served as prototype for the development of numerical algorithms. Therefore, an accurate prediction of the flow structure and the heat transfer in such configuration is of great interest. However, due to the complex behaviour exhibit (see Figure 1), and despite the great effort devoted, an accurate turbulence modelling of this configuration remains as a great challenge. On the other hand, direct numerical simulation (DNS) has become an essential tool to give new insights into the physics of turbulence and provide indispensable data for the development of better turbulence models. However, at high Ra-numbers, DNS simulations are not feasible.

In this context, a dynamically less complex mathematical formulation is sought. In the quest for such a formulation, we consider regularizations (smooth approximations) of the nonlinear convective term. The first outstanding approach in this direction goes back to Leray [1]; the Navier-Stokes- $\alpha$ model also forms an example of regularization modelling (see [2], for instance). The regularization methods basically alter the convective terms to reduce the production of small scales of motion. In doing so, we proposed to preserve the symmetry and conservation properties of the convective terms exactly. This requirement yielded a family of symmetry-preserving regularization models [3]: a novel class of regularizations that restrain the convective production of smaller and smaller scales of motion in an unconditional stable manner, meaning that the velocity cannot blow up in the energy-norm (in 2D also: enstrophy-norm). In this work, a method to dynamically determine the regularization parameter (local filter length) with the requirement that the vortex-stretching must be stopped at the scale set by the grid is also proposed and tested. The numerical algorithm used to solve the governing equations preserves the conservation properties too [4] and is therefore well-suited to test the proposed simulation model.

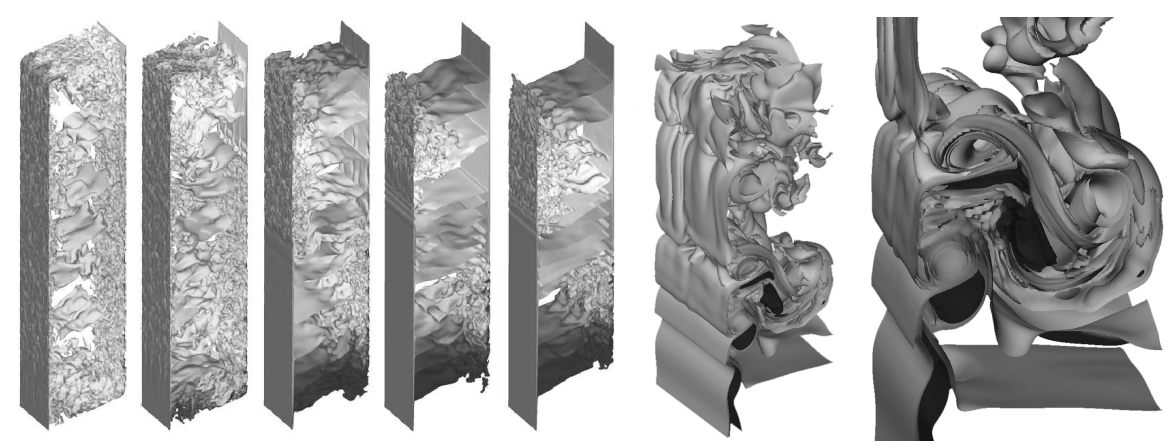

Fig. 1 Left: several instantaneous temperature fields at $R a=10^{11}$ and $P r=0.71$ (air). Right: zoom around the point where the vertical boundary layer becomes totally disrupted and large eddies are ejected. 


\section{$2 \mathscr{C}_{4}$-regularization modelling}

We restrict ourselves to the $\mathscr{C}_{4}$ approximation (see [3], for details): the convective term in the Navier-Stokes equations is then replaced by the following $\mathscr{O}\left(\varepsilon^{4}\right)$ accurate smooth approximation $\mathscr{C}_{4}(u, v)$ given by

$$
\mathscr{C}_{4}(u, v)=\mathscr{C}(\bar{u}, \bar{v})+\overline{\mathscr{C}\left(\bar{u}, v^{\prime}\right)}+\overline{\mathscr{C}\left(u^{\prime}, \bar{v}\right)}
$$

where $\mathscr{C}(u, v)=(u \cdot \nabla) v$ represents the convective operator. Note that here a prime indicates the residual of the filter, e.g. $u^{\prime}=u-\bar{u}$, which can be explicitly evaluated, and $\overline{(\cdot)}$ represents a normalised self-adjoint linear filter with filter length $\varepsilon$. Therefore, the governing equations result to

$$
\partial_{t} u+\mathscr{C}_{4}(u, u)=\frac{1}{R e} \Delta u-\nabla p+f ; \quad \nabla \cdot u=0
$$

Note that the $\mathscr{C}_{4}$ approximation is also a skew-symmetric operator like the original convective operator. Hence, the same inviscid invariants -kinetic energy, enstrophy in 2D and helicity- that the original Navier-Stokes equations are kept for the new set of partial differential equations to be solved (2).

Since now the performance of the $\mathscr{C}_{4}$ approximation has been successfully tested for a turbulent channel flow [3] and an air-filled $(\operatorname{Pr}=0.71)$ differentially heated cavity (DHC) of height aspect ratio $A=4$ at $R a$-numbers (based on the cavity height) $10^{10}[5,6]$ and $10^{11}$ [7] by means of direct comparison with the DNS results $[8,9,10]$. In the latter cases, the filter width $\varepsilon$ was treated as a parameter. Then, the value of $\varepsilon$ needed to be prescribed in advanced. Figure 2 displays illustrative results for the aforesaid DHC problem at $R a=10^{10}$ as a function of $\varepsilon / h$ ratio. These results show that the numerical solution is weakly dependent for sufficiently large values of $\varepsilon$. Similar behaviour has also been observed for the other abovementioned configurations.
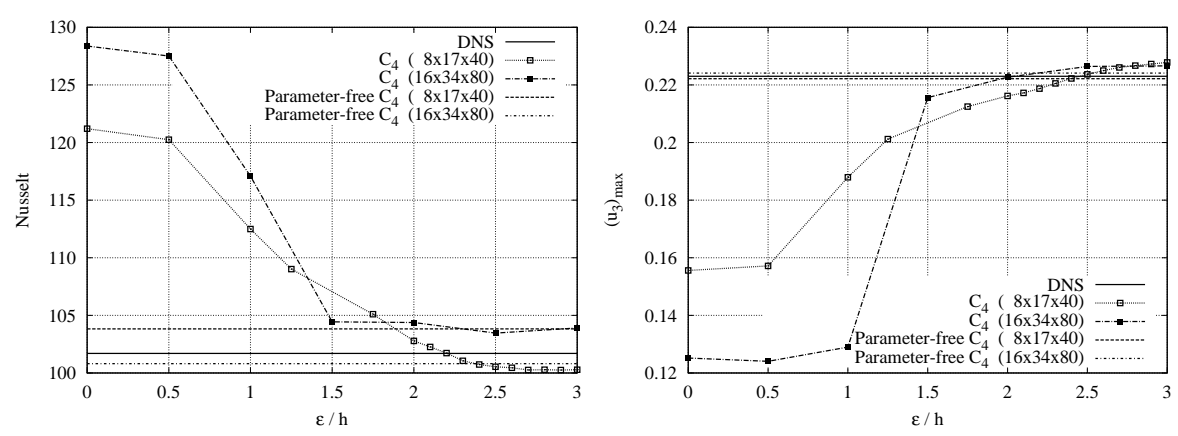

Fig. 2 The overall averaged Nusselt number (left) and the maximum of the averaged vertical velocity at the horizontal mid-height plane (right) as a function of the ratio of the filter length $\varepsilon$ to the average grid width $h$. 


\section{On the dynamic determination of the filter length}

In the present work, we propose to determine $\varepsilon$ dynamically with the requirement that the vortex-stretching must be stopped at the scale set by the grid. The idea behind is to modify convective operator sufficiently to guarantee that the following inequality is hold

$$
\lambda_{k}(\varepsilon)-v k^{2} \leq 0
$$

where $\lambda_{k}(\varepsilon)=\omega_{k} \cdot \mathscr{C}_{4}(\omega, u) /\left(\omega_{k} \cdot \omega_{k}\right)$ is the Rayleigh quotient of the vortexstretching at the grid scale, $k=\pi / h$. In practice, the value of $\lambda_{k}$ has been bounded by the largest (positive) eigenvalue of the straintensor $S$,

$$
\lambda_{k}(\varepsilon) \leq f_{4, k}(\varepsilon) \lambda_{\max }(S)
$$

For the $\mathscr{C}_{4}$-approximation, the damping function, $0<f_{4, k} \leq 1$, at the highest frequency is given by $3 \hat{g}_{k}^{2}(\varepsilon)-2 \hat{g}_{k}^{3}(\varepsilon)$ (see [3], for details), where $0<\hat{g}_{k}(\varepsilon) \leq 1$ is the transfer function of the linear filter. Therefore, it suffices that the following inequality be locally hold

$$
3 \hat{g}_{k}^{2}(\varepsilon)-2 \hat{g}_{k}^{3}(\varepsilon) \leq \frac{v k^{2}}{\lambda_{\max }(S)} \quad \longrightarrow \quad \hat{g}_{k}(\varepsilon) \quad \longrightarrow \quad \varepsilon
$$

to guarantee that the vortex-stretching mechanism be stopped at the smallest scale set by the mesh.

\begin{tabular}{l|c|cc} 
& DNS & RM1 & RM2 \\
\hline$R a=10^{10}$ & $128 \times 190 \times 462$ & $16 \times 34 \times 80$ & $8 \times 17 \times 40$ \\
$R a=10^{11}$ & $128 \times 682 \times 1278$ & $12 \times 45 \times 85$ & $8 \times 30 \times 56$
\end{tabular}

Table 1 Description of meshes: the spanwise $\left(N_{x}\right)$, the wall-normal horizontal $\left(N_{y}\right)$, and the vertical $\left(N_{z}\right)$ resolutions for tested cases. Further details about the meshing can be found in [8].

\section{Results for a turbulent differentially heated cavity}

The performance of the proposed method to dynamically determine the regularization parameter $\varepsilon$ of the $\mathscr{C}_{4}$ approximation has also been tested for the aforesaid DHC problem. Again two very coarse meshes (see table 1) have been solved. In this work, a fourth-order accurate Gaussian filter [11] has been chosen. In 1D it becomes

$$
\bar{u}_{i}=\frac{\varepsilon^{4}-4 \varepsilon^{2}}{1152}\left(u_{i+2}+u_{i-2}\right)+\frac{16 \varepsilon^{2}-\varepsilon^{4}}{288}\left(u_{i+1}+u_{i-1}\right)+\frac{\varepsilon^{4}-20 \varepsilon^{2}+192}{192} u_{i}
$$


where the filter length, $\varepsilon$, need not be restricted to integers multiples of the grid width. The boundary conditions that supplement the Navier-Stokes equations are also applied to (6).

First results displayed in Figure 2 exhibit the great potential of this method. Note that in the parameter-free $\mathscr{C}_{4}$ cases, the results do not depend on $\varepsilon / h$. At least as good results as the optimal $\varepsilon / h$ ratio determined by trial-and-error procedure are being obtained.

\subsection{Grid dependence analysis}

A reliable modelling of turbulence at (very) coarse grids is a great challenge. The coarser the grid, more convincing model quality is perceived. However, it might happen that solution be strongly dependant on meshing parameters and thus some particular combinations could 'accidentally' provide good results. An example of this behaviour can be found in [12] for a turbulent channel flow. In order to elucidate this point, the same DHC problem has been solved on a series of 50 randomly generated meshes: with $\left(N_{x}, N_{y}, N_{z}\right)$-values limited by those given by meshes RM1 and RM2 (see Table 1), i.e. $8 \leq N_{x} \leq 16,17 \leq N_{y} \leq 34$, and $40 \leq N_{z} \leq 80$. Results for the overall Nusselt and the centerline stratification values are displayed in Figure 3. At first sight, we can observe that the parameter-free $\mathscr{C}_{4}$ modelling predicts good results irrespective of the meshing whereas very poor and dispersed results are obtained when the model is switched off. Especially significant is the fairly good prediction of the stratification (note the large dispersion obtained without model).

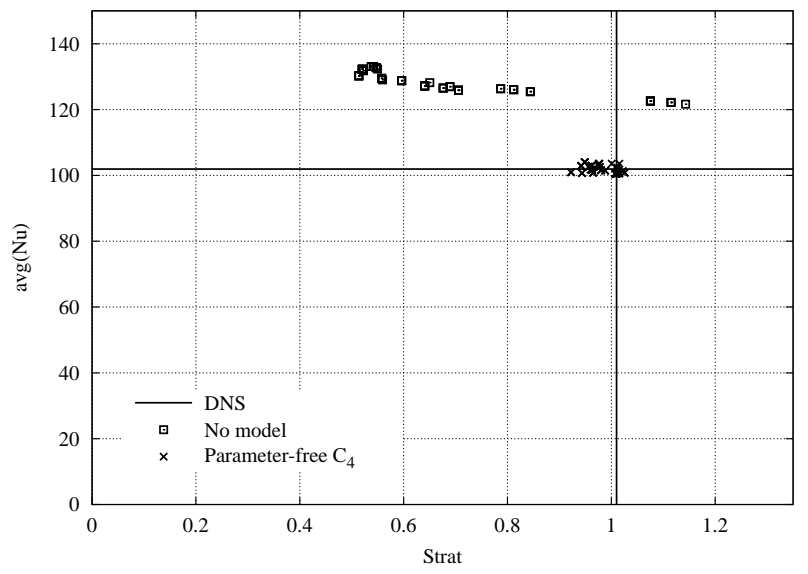

Fig. 3 The overall Nusselt number and the centerline stratification for randomly generated grids. 


\subsection{Performance at higher Ra-numbers}

The performance of the parameter-free $\mathscr{C}_{4}$-regularization model has also been tested at a higher $R a$-number $\left(R a=10^{11}\right)$. Results for two coarse meshes (see Table 1) are displayed in Figure 4 and compared with the DNS reference results $[9,10]$. Although in this case the Ra-number is one order of magnitude higher and the mesh resolutions are kept almost the same respect to the case at $R a=10^{10}$, the proposed method is able to predict reasonable well the mean flow values.
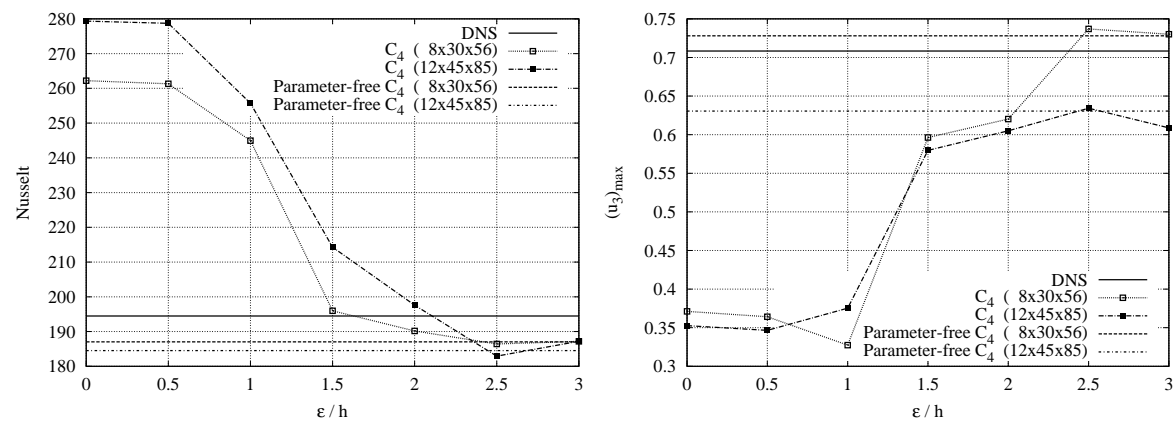

Fig. 4 The overall averaged Nusselt number (left) and the maximum of the averaged vertical velocity at the horizontal mid-height plane (right) as a function of the ratio of the filter length $\varepsilon$ to the average grid width $h$ at $R a=10^{11}$.

\section{Conclusions}

The performance of the proposed method to dynamically determine the regularization parameter $\varepsilon$ of the $\mathscr{C}_{4}$ approximation has been tested for an air-filled DHC problem of aspect ratio 4 and Rayleigh numbers $10^{10}$ and $10^{11}$. Results presented here exhibit the great potential of the proposed method as new turbulence model: the parameter-free $\mathscr{C}_{4}$ approach is able to predict at least as good results as the optimal $\varepsilon / h$ ratio determined by trial-and-error procedure.

In conclusion, the proposed method constitutes a parameter-free turbulence model. Moreover, since no ad hoc phenomenological arguments that cannot be formally derived for the Navier-Stokes equations are used it suggest that this method should be valid for other configurations. Nevertheless, more simulations for a wide variety of cases and meshes will be necessary to confirm these conclusions. 


\section{Acknowledgements}

This work has been financially supported by the Ministerio de Educación y Ciencia, Spain, (Project: "Estudio y parametrización de fenómenos de transferéncia de calor y dinámica de fluidos involucrados en diseños de arquitectura bioclimática". Contract/grant number ENE2006-14247) and a postdoctoral fellowship Beatriu de Pinós (2006 BP-A 10075) by the Generalitat de Catalunya.

\section{References}

1. J. Leray. Sur le movement d'un liquide visqueaux emplissant l'espace. Acta Mathematica, 63:193-248, 1934.

2. B. J. Geurts and D. D. Holm. Regularization modeling for large-eddy simulation. Physics of Fluids, 15:L13-L16, 2003.

3. Roel Verstappen. On restraining the production of small scales of motion in a turbulent channel flow. Computers and Fluids, 37:887-897, 2008.

4. R. W. C. P. Verstappen and A. E. P. Veldman. Symmetry-Preserving Discretization of Turbulent Flow. Journal of Computational Physics, 187:343-368, 2003.

5. F. X. Trias, M. Soria, A. Oliva, and R. W. C. P. Verstappen. Regularization models for the simulation of turbulence in a differentially heated cavity. In Proceedings of the European Computational Fluid Dynamics Conference (ECCOMAS CFD 2006), Egmond aan Zee, The Netherlands, September 2006.

6. F. X. Trias, R. Verstappen, M. Soria, and A. Oliva. Symmetry-preserving regularization modelling of a turbulent differentially heated cavity. In LES in Science and Technology, Poznan, Poland, April 2008.

7. F. X. Trias, R. W. C. P. Verstappen, M. Soria, A. Gorobets, and A. Oliva. Regularization modelling of a turbulent differentially heated cavity at $R a=10^{11}$. In 5th European ThermalSciences Conference, EUROTHERM 2008, Eindhoven, The Netherlands, May 2008.

8. F. X. Trias, M. Soria, A. Oliva, and C. D. Pérez-Segarra. Direct numerical simulations of twoand three-dimensional turbulent natural convection flows in a differentially heated cavity of aspect ratio 4. Journal of Fluid Mechanics, 586:259-293, 2007.

9. F. X. Trias, A. Gorobets, M. Soria, and A. Oliva. Direct numerical simulation of a differentially heated cavity of aspect ratio 4 with $R a$-number up to $10^{11}$ - Part I: Numerical methods and time-averaged flow. International Journal of Heat and Mass Transfer, 53:665-673, 2010.

10. F. X. Trias, A. Gorobets, M. Soria, and A. Oliva. Direct numerical simulation of a differentially heated cavity of aspect ratio 4 with $R a$-number up to $10^{11}$ - Part II: Heat transfer and flow dynamics. International Journal of Heat and Mass Transfer, 53:674-683, 2010.

11. P. Sagaut and R. Grohens. Discrete Filters for Large Eddy Simulations. International Journal for Numerical Methods in Fluids, 31:1195-1220, 1999.

12. J. Meyers and P. Sagaut. Is plane-channel flow a friendly case for the testing of large-eddy simulation subgrid-scale models? Physics of Fluids, 19:048105, 2007. 